\title{
MRI Signatures of Brain Macrostructural Atrophy and Microstructural Degradation in Frontotemporal Lobar Degeneration Subtypes
}

\author{
Yu Zhang ${ }^{\mathrm{a}, \mathrm{b}, *}$, Maria Carmela Tartagliaa ${ }^{\mathrm{c}, \mathrm{d}}$, Norbert Schuff ${ }^{\mathrm{a}, \mathrm{b}}$, Gloria C. Chiang ${ }^{\mathrm{a}, \mathrm{b}}$, \\ Christopher Ching ${ }^{\mathrm{a}, \mathrm{b}}$, Howard J. Rosen ${ }^{\mathrm{c}}$, Maria Luisa Gorno-Tempini ${ }^{\mathrm{c}}$, Bruce L. Miller \\ and Michael W. Weiner ${ }^{\mathrm{a}, \mathrm{b}}$ \\ ${ }^{a}$ Department of Veterans Affairs Medical Center, Center for Imaging of Neurodegenerative Diseases, \\ San Francisco, CA, USA \\ ${ }^{\mathrm{b}}$ Department of Radiology, University of California, San Francisco, CA, USA \\ ${ }^{\mathrm{c}}$ Department of Neurology, Memory and Aging Center, University of California, San Francisco, CA, USA \\ ${ }^{\mathrm{d}}$ University Health Network and Tanz Centre for Research in Neurodegenerative Disease, \\ University of Toronto, Toronto, ON, Canada
}

Handling Associate Editor: J. Wesson Ashford

Accepted 4 August 2012

\begin{abstract}
Brain magnetic resonance imaging (MRI) studies have demonstrated regional patterns of brain macrostructural atrophy and white matter microstructural alterations separately in the three major subtypes of frontotemporal lobar degeneration (FTLD), which includes behavioral variant frontotemporal dementia (bvFTD), semantic dementia (SD), and progressive nonfluent aphasia (PNFA). This study was to investigate to what extent the pattern of white matter microstructural alterations in FTLD subtypes mirrors the pattern of brain atrophy, and to compare the ability of various diffusion tensor imaging (DTI) indices in characterizing FTLD patients, as well as to determine whether DTI measures provide greater classification power for FTLD than measuring brain atrophy. Twenty-five patients with FTLD (13 with bvFTD, 6 with SD, and 6 with PNFA) and 19 healthy age-matched control subjects underwent both structural MRI and DTI scans. Measurements of regional brain atrophy were based on T1-weighted MRI data and voxel-based morphometry. Measurements of regional white matter degradation were based on voxelwise as well as regions-of-interest tests of DTI variations, expressed as fractional anisotropy, axial diffusivity, and radial diffusivity. Compared to controls, bvFTD, SD, and PNFA patients each exhibited characteristic regional patterns of brain atrophy and white matter damage. DTI overall provided significantly greater accuracy for FTLD classification than brain atrophy. Moreover, radial diffusivity was more sensitive in assessing white matter damage in FTLD than other DTI indices. The findings suggest that DTI in general and radial diffusivity in particular are more powerful measures for the classification of FTLD patients from controls than brain atrophy.
\end{abstract}

\footnotetext{
*Correspondence to: Yu Zhang, MD, Center for Imaging of Neurodegenerative Diseases, VA Medical Center, 4150, Clement Street, San Francisco, CA 94121, USA. Tel.: +1 415 2214810; Ext: 2158; Fax: +1 415 6682864; E-mail: Yu.Zhang@ucsf.edu.
} 
Keywords: Behavioral variant frontotemporal dementia, diffusion tensor imaging, frontotemporal lobar degeneration, multimodality MRI, progressive nonfluent aphasia, semantic dementia

Supplementary data available online: http://dx.doi.org/10.3233/JAD-2012-121156

\section{INTRODUCTION}

Behavioral variant frontotemporal dementia (bvFTD), semantic dementia (SD), and progressive nonfluent aphasia (PNFA) are the three main clinical subtypes of frontotemporal lobar degeneration (FTLD), a class of progressive neurodegenerative diseases, affecting primarily frontal and temporal lobe regions [1]. Each of these FTLD subtypes displays a unique clinical phenotype associated with a characteristic pattern of morphological abnormalities in the brain. bvFTD, also referred to as frontal variant FTD, features a prominent behavioral component that includes changes in personality often associated with disinhibition, apathy, and loss of empathy. On structural magnetic resonance imaging (MRI), bvFTD shows frontal and/or anterior temporal lobe atrophy of both gray and white matter [2-7]. On the other hand, patients with $\mathrm{SD}$, also referred to as temporal variant FTD, or semantic variant primary progressive aphasia, exhibit impairment in word and object meaning, and have gray and white matter atrophy in the temporal lobes, predominantly in the left brain hemisphere [8-13]. Lastly, patients with PNFA, also referred to as nonfluent variant primary progressive aphasia, present clinically with nonfluent, effortful, agrammatic speech and atrophy in the left perisylvian area, including inferior frontal operculum, dorsal anterior insular regions, and expanding to premotor and supplementary motor areas [8, 14, 15] on MRI.

In addition to brain atrophy, diffusion tensor imaging (DTI), which is sensitive to microstructural alterations in white matter and allows in vivo reconstruction of white matter tracts based on the directional diffusion properties of water, has also revealed differential white matter abnormalities across FTLD subtypes. In particular, patients with bvFTD showed on DTI abnormalities in the frontal and temporal white matter [16-20], involving predominantly the anterior and posterior corpus callosum, uncinate fasiculus, and arcuate fasciculus (a subcomponent of the superior longitudinal fasciculus). In contrast to bvFTD, SD patients showed DTI alterations predominantly in temporal brain regions, including the uncinate fasciculus, inferior longitudinal fasciculus, as well as the inferior anterior corpus callosum [16, 20-23]. Recent DTI studies in PNFA reported abnormal DTI in the superior longitudinal fasciculus [18, 20, 21]. Replicating these findings with different samples of FTLD patients may prove the importance of DTI measurement to further research aims.

A complicating debate is the ambiguity of DTI as to its biological underpinning. In general, fractional anisotropy (FA), a summary index of DTI commonly used to express diffusion directionality, is believed to reflect various aspects of axonal injury, including demyelination, gliosis, and gross axonal degeneration [24]. On the other hand, growing evidence from animal studies suggests that alternative DTI metrics such as radial diffusivity (DR, the average of the two smaller eigenvalues of the diffusion tensor) and axial diffusivity (DA, the largest eigenvalue of the tensor) are more specific indices of the biological underpinnings, although fundamental limitations remain. Specifically, increased DR has been associated primarily with severity of demyelination [25]. Although in most previous studies of FTLD [18-23, 26], increased diffusivities (DR and DA) and reduced FA were shown broadly concordant and anatomically consistent, it is still unclear whether the absolute diffusivities are more valuable than FA in characterizing FTLD patients. Furthermore, despite a growing body of structural MRI and DTI studies in FTLD [18, 20, 22], little is known about the relationships and differences between regional macrostructural brain atrophy and microstructural diffusion changes in contributing the FTLD diagnosis. Moreover, whether atrophy or diffusion measures provide a better characterization of FTLD is a matter of intense debate.

In this study, DTI and structural MRI data were obtained in the same patients and control subjects for direct comparisons. The main goals of this study were threefold: first, to determine the extent to which the pattern of white matter microstructural alterations in FTLD subtypes mirrors the pattern of brain atrophy; second, to compare the ability of various DTI indices to characterize FTLD patients; and third, to determine whether DTI measures provide more accurate classification of FTLD patients than MRI measures of brain atrophy. 
Table 1

Summary of the demographic and structural MRI variables

\begin{tabular}{|c|c|c|c|c|}
\hline & $\mathrm{CN}$ & bvFTD & SD & PNFA \\
\hline Number & 19 & 13 & 6 & 6 \\
\hline Age & $63.1 \pm 7.6$ & $59.5 \pm 6.5$ & $64.8 \pm 5.8$ & $64.3 \pm 5.0$ \\
\hline Gender $(\mathrm{M}: \mathrm{F})$ & $10: 9$ & $10: 3$ & $4: 2$ & $1: 5$ \\
\hline Handedness & $16 \mathrm{R}: 1 \mathrm{~L}: 2 \mathrm{~A}$ & $11 \mathrm{R}: 1 \mathrm{~L}$ & 4R: 1L: 1A & $6 \mathrm{R}$ \\
\hline Education (y) & $17.1 \pm 2.6$ & $15.5 \pm 3.3$ & $15.5 \pm 1.9$ & $14.8 \pm 2.7$ \\
\hline Durations of symptom (y) & - & $6.1 \pm 4.7$ & $6.9 \pm 2.4$ & $4.2 \pm 1.4^{\mathrm{b}}$ \\
\hline MMSE & $29.6 \pm 0.5$ & $25.8 \pm 3.8^{\mathrm{a}}$ & $26.2 \pm 4.8^{\mathrm{a}}$ & $27.2 \pm 1.1^{\mathrm{a}}$ \\
\hline CDR & 0 & $1.04 \pm 0.3^{\mathrm{a}}$ & $0.7 \pm 0.4^{\mathrm{a}}$ & $0.5 \pm 0.4^{\mathrm{a}, \mathrm{b}}$ \\
\hline brain size (TIV) (cc) & $1536.6 \pm 177.8$ & $1634.9 \pm 235.2$ & $1643.5 \pm 264.2$ & $1610.1 \pm 192.2$ \\
\hline Gray matter volume/TIV & $0.45 \pm 0.03$ & $0.43 \pm 0.02$ & $0.43 \pm 0.01$ & $0.42 \pm 0.01$ \\
\hline White matter volume/TIV & $0.28 \pm 0.02$ & $0.27 \pm 0.02$ & $0.27 \pm 0.03$ & $0.28 \pm 0.01$ \\
\hline
\end{tabular}

${ }^{a}$ Significant differences between patients and control group by ANOVA tests.

bSignificant differences among FTLD subtypes.

Handedness: L, left; R, right; A, ambidextrous; Handedness of one bvFTD patient is unknown. TIV, total intracranial volume.

\section{MATERIALS AND METHODS}

\section{Subjects}

Twenty-five patients with FTLD (13 bvFTD, 6 SD, and 6 PNFA) and 19 age-matched healthy control (CN) subjects were included in this MRI study. A summary of the subject demographics and relevant clinical information is listed in Table 1. All patients were recruited from the Memory and Aging Center of the University of California, San Francisco. Diagnosis and sub-grouping were based on published research criteria [27]. In addition, the patients had the following: (1) Clinical Dementia Rating (CDR) and Mini Mental State Examination (MMSE) completed within 90 days of the scan; (2) absence of any comorbid motor neuron disease; (3) no moderate to severe white matter hyperintensities (i.e., $>3 \mathrm{~mm}$ periventricular hyperintensities, or $>10 \mathrm{~mm}$ deep white matter lesions, or $>10$ lesions, on T2-weighted images); (4) no logopenic variant primary progressive aphasia patients were included in the study due to the small number of these patients; and (5) no other major neurological conditions such as those diagnosed with other types of dementia (Alzheimer's disease, vascular dementia, Lewy bodies), or psychiatric illness (anxiety, depression), or other neurodegenerative disorders (Huntington's disease, multiple system atrophy), strokes, epilepsy, brain tumors and other systemic diseases that affect brain function. All subjects or their legal guardians gave written informed consent before participating in the study, which was approved by the Committees of Human Research at the University of California at San Francisco and the Veterans Affairs Medical Center.

\section{Data acquisition}

All scans were performed on a 4 Tesla (Bruker/Siemens) MRI system with a single housing birdcage transmit and 8 channel receive coil. T1-weighted images were obtained using a 3D volumetric magnetization prepared rapid gradient echo (MPRAGE) sequence with TR/TE/TI = 2300/3/950 ms, a 7-degree flip angle, and $1.0 \times 1.0$ $\times 1.0 \mathrm{~mm}^{3}$ resolution. In addition, FLAIR (fluid attenuated inversion recovery) images with TR/TE/TI = $5000 / 355 / 1900 \mathrm{~ms}$ were acquired to identify white matter lesions and to improve brain extraction in the segmentation procedure. DTI was acquired using a twice-refocused spin-echo diffusion echo-planar imaging (EPI) sequence supplemented with twofold parallel imaging acceleration (GRAPPA) to reduce susceptibility distortions. DTI imaging parameters were TR/TE $=6000 / 77 \mathrm{~ms}$, field of view $256 \times 224 \mathrm{~cm}$, $128 \times 112$ matrix size, yielding $2 \times 2 \mathrm{~mm}^{2}$ in-plane resolution, 40 slices each $3 \mathrm{~mm}$ thick. One reference image $(b=0)$ and six diffusion-weighted images ( $b=800 \mathrm{~s} / \mathrm{mm}^{2}$, each along 6 non-collinear directions) were acquired. Maps of FA, DA, and DR were reconstructed after eddy-current corrections [28].

\section{Structural image (Tl-weighted MRI) processing}

The assessment of gray and white matter volume variations were performed using SPM8 software (http: //www.fil.ion.ucl.ac.uk/spm/software/spm8/) based on an "optimized" voxel-based morphometry (VBM) procedure, which in detail included several steps: (1) Tissue segmentation: An Expectation 
Maximization Segmentation (EMS) tool (https:// mirc.uzleuven.be/downloads/ems.php) was used to obtain probabilistic maps of gray matter, white matter, and cerebrospinal fluid (CSF) from the T1-weighted MRI data. A binary brain mask derived from the FLAIR image was used to exclude meninges and major blood vessels. (2) Creation of populationbased gray and white matter templates: After tissue segmentation, gray and white matter probabilistic maps of all subjects were initially transformed into the standard MNI (Montreal Neurological Institute) space. Population-based gray and white matter templates were then created by averaging the gray and white matter probabilistic maps of all subjects. Population-based templates provide generally more accurate results for spatial normalization of the images from different subjects than standard templates, which usually are derived from a younger population than the one studied here. (3) Spatial normalization: To achieve high anatomical correspondence across the brain images from different subjects, the segmented gray and white matter probabilistic maps in their respective native space were spatially normalized again to the population-based gray and white matter templates using a nonlinear transformation with 16 iterations. (4) Jacobian modulation: The spatially normalized gray and white images were multiplied by the Jacobian determinants of the transformation (modulation) to obtain volume differences. (5) Smoothing: The modulated gray and white matter images were smoothed with a $10 \mathrm{~mm}^{3}$ full-width-at-half-maximum (FWHM) Gaussian kernel to reduce variations from mis-registrations and to perform voxel-wise statistics.

\section{DTI image processing}

The assessment of regional DTI variations including FA, DR, and DA was also performed voxelwise within the framework of SPM8. In brief, the DTI images in native space underwent the following procedures: (1) Creation of a population-based FA template: An averaged FA image was first created from all subjects' FA images that were transformed initially to the EPI-derived MNI template in SPM. Details of the transformation procedures were reported in a previous publication [19]. (2) Spatial normalization and smoothing: The FA image of each subject in their native space was recursively aligned to the population-based FA template using nonlinear transformation. These spatially normalized FA images were smoothed with a $4 \mathrm{~mm}^{3}$ FWHM Gaussian kernel.

\section{Voxel-wise statistical analyses}

Comparisons between each FTLD subtype and control group were tested voxel-by-voxel using twosample $t$-tests with diagnosis as the main effect. Age, gender, and years of education were included in the tests as nuisance variables. In addition, total intracranial volume (TIV), which had been obtained automatically by the SPM8 segmentation tool, was used as a covariate in tests for differences in structural volume to account for variations in head size. To reduce variations due to voxel heterogeneity, e.g., varying amounts of gray matter, white matter, and CSF within voxels, a lower threshold of $85 \%$ brain tissue fraction was applied for voxels to reduce contributions from CSF. Similarly, a lower threshold of $\mathrm{FA}=0.20$ was applied to reduce contributions from gray matter (which usually has small FA values) and also to reduce measurement bias toward higher diffusion anisotropy (due to the Riccian noise characteristic of DTI). Only voxels that all subjects had in common were included in the statistical analysis. The resulting statistical parametric maps were displayed with significant voxel-levels of $p=0.001$, uncorrected for multiple comparison, and with significant clusterlevels of $p=0.05$, corrected for family wise error (FWE).

\section{Statistical analyses of ROIs derived measures}

The relationship between brain volume and DTI measures in contributing to group classification was assessed in regions of interest (ROIs). These ROIs were derived from the statistical maps of the voxelwise analysis as follows: for each gray or white matter volumetric measurement and DTI measures of FA, DR, and DA, ROIs were determined based on the largest cluster that was shown on each statistical map of paired-group comparison, thus there were three groups of ROIs (ROI_1, ROI_2, and ROI_3) determined by the three paired-group comparisons. These voxels in each ROI were assigned onto the structural or DTI images that were normalized in the standard space to obtain regional gray or white matter volumes, and DTI values for each subject. Statistical analysis of ROIs from the volumetric and DTI measurement was performed using $\mathrm{R}$ (the R project of statistical computing: http://www.rproject.org/). Variations of volumetric and DTI (FA, DR, and DA) of each ROI were analyzed separately as a linear function of diagnosis with adjustments for age and gender. Two models were fitted by maximum likelihood: the first model included only the covariates, 
while diagnosis was added in the second model. The resulting fits were compared sequentially via $F$-tests to ensure that diagnosis added explanatory power to the base model. To determine the DTI measures that best characterize FTLD patients (the second aim), nested logistic regression models, in which all DTI ROIs predicted group classification were built and compared with models in which one ROI was removed at a time. Quality of classifications was measured using the Matthews correlation coefficient (MCC), which takes into account true and false positives and negatives while providing generally a balanced assessment even if the classes have different sizes [29]. An MCC of value +1 represents a perfect prediction, zero is an average random prediction, and -1 represents an inverse prediction. To further assess the variability in predictions, cross-validation was performed on the frequency of classification errors. To determine whether diffusion measures or atrophy measures achieve better classification of FTLD patients (the third aim), logistic regressions were performed and classification accuracy was evaluated in terms of sensitivity and specificity based on the area under the curve (AUC) of a receiver operator characteristic analysis and augmented by 4 -fold cross-validation with boot-strap to control for overfitting. These logistic regression tests were performed between control and the FTLD patient group with all three subtypes combined. The statistical significance of differences in MCC and AUC tests between FTLD patients and control subjects was evaluated using Wilcoxon rank-sum tests. The significant thresholds of all ROI tests were set with multiple comparison (False Discovery Rate, FDR)-adjusted alpha level of 0.05 .

\section{Supplementary tract-specific DTI analysis}

To eliminate the possibility of spurious findings in voxelwise tests due to image mis-registration, we also performed a supplementary tract-specific DTI analysis for each individual in the respective native DTI space using dTV and Volume-one software package (http://www.ut-radiology.umin.jp/ people/masutani/dTV.htm) with fiber assignments based on continuous tracking (FACT) approach to achieve a three-dimensional tract reconstruction. The details of individual fiber tracking and the determinations of tracts of interest (TOI) are described in our previously published paper [19]. The final TOI regions included: anterior corpus callosum (a.CC) and posterior corpus callosum (p.CC) derived from the anterior and posterior halves of the callosal fibers covering a $10 \mathrm{~mm}$ thickness of the mid-sagittal slices, bilateral anterior cingulum and posterior cingulum derived from the anterior and posterior halves of the cingulum fiber, bilateral parahippocampal cingulum, uncinate fasciculi, arcuate fasciculi, and the fornix. Differences between each FTLD subtype and CN were tested separately for each measurement of FA, DA, and DR in the listed TOI regions using linear regression models that described above to determine the group difference which is contributed by diagnosis in addition to age and gender. To evaluate which DTI measure most accurately characterizes FTLD patients, logistic regression augmented by 4-fold cross-validation and bootstrap was performed between control and the overall FTLD patients and classification accuracy quantified using an operator characteristic analysis and the area under the ROC curve (AUC). For each DTI measure, all TOIs were simultaneously included in the logistic regression model. The differences between AUCs of each DTI measure were evaluated using Wilcoxon rank-sum tests. The significance threshold of the above tests was set at FDR-adjusted alpha level of 0.05 .

\section{RESULTS}

\section{Demographic, clinical, and cognitive data}

As summarized in Table 1, there were no significant differences between the groups with respect to age, gender, handedness, and years of education. It should be noted that although there was a trend of greater proportion of females in the PNFA group as comparing with the bvFTD group, adding gender into the statistical models of ROI analyses did not alter the significant differences between the groups. FTLD patients overall had significantly worse MMSE and CDR scores compared to $\mathrm{CN}$, as expected. However, MMSE scores were not significantly different among the FTLD subtypes. PNFA patients had lower ( $p=0.01$, ANOVA) CDR scores than patients with bvFTD. PNFA patients also had a shorter $(p=0.04$, ANOVA) duration of symptoms than SD patients, but not significantly shorter compared to the bvFTD patients. There was no significant difference between FTLD subtypes and $\mathrm{CN}$ with regards to the TIV, global gray and white matter volumes.

\section{Voxel-wise comparisons between bvFTD and CN}

Figure 1 shows the regional patterns of significant gray matter atrophy (1st row), white matter atrophy 


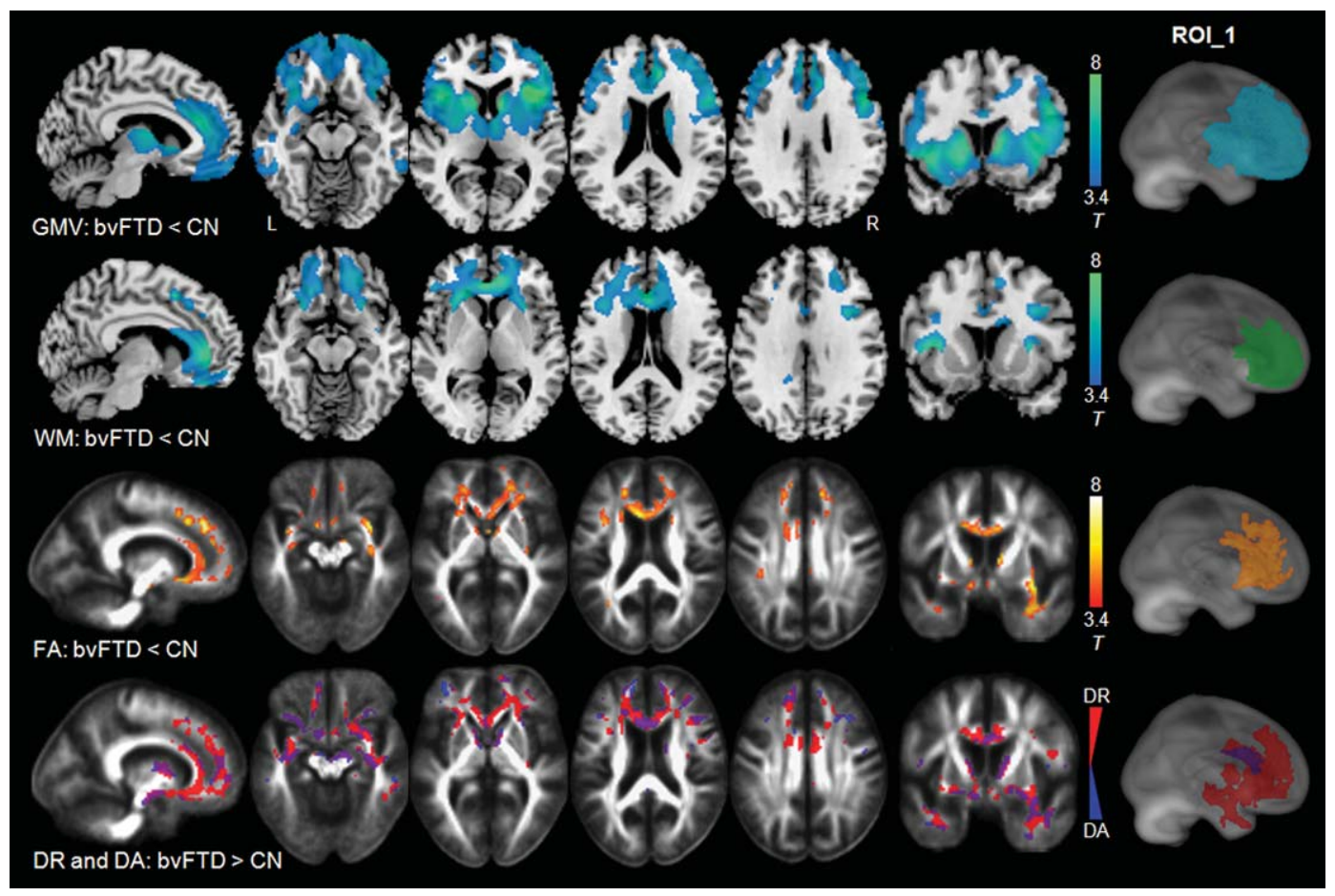

Fig. 1. Comparison between bvFTD and CN, row 1-2: regions of significant gray and white matter atrophy (in blue-green color) superimposed on the T1-weighted image; row 3: regions of significant FA reduction (in red-yellow color) superimposed on the FA template; row 4: regions of significantly increased DR (in red color) and increased DA (in blue color) superimposed on the FA template. Statistical map-derived ROI was determined by the largest-sized cluster that showed significant $(p=0.001)$ on each paired-group comparison. ROI_1 indicates the anatomical locations of these statistical map-derived ROIs superimposed on a rendered brain.

(2nd row), FA reduction (3rd row), and an overlay of increased DR and increased DA from separate analyses (4th row) in patients with bvFTD compared to $\mathrm{CN}$. Compared to $\mathrm{CN}$, bvFTD patients showed extensive gray matter atrophy in bilateral frontal and temporal regions including anterior cingulate gyri, striatal, and frontopolar regions and most pronounced in bilateral frontoinsular regions. White matter atrophy was observed predominantly in the frontal areas including bilateral medial, inferior, middle, and superior frontal white matter. The most pronounced white matter atrophy was seen in the anterior callosal region. In contrast to the structural changes, patients with bvFTD showed FA reductions bilaterally in the frontal and temporal white matter, compared to $\mathrm{CN}$. Regional patterns of increases in DR (in red color) were similar while more widespread than the pattern of FA reduction. Increases in DA (in blue color) were observed primarily in concomitant regions with increases in DR (in purple color), including the anterior callosal region, fornix, bilateral uncinate, and some extra-fiber regions.
The anterior cingulate regions, however, showed bilaterally increased DR without significant changes in DA.

\section{Voxel-wise comparisons between SD and CN}

Figure 2 illustrates the regional patterns of significant gray matter atrophy (1st row), white matter atrophy (2nd row), FA reduction (3rd row), and an overlay of increased DR and increased DA (4th row) in patients with SD compared to $\mathrm{CN}$. Compared to $\mathrm{CN}$, SD patients showed a characteristic pattern of gray and white matter atrophy in both temporal lobes, predominantly in the left temporal pole. FA reductions in patients with SD were observed in bilateral uncinate fasciculi and in the left temporal white matter regions including the left anterior part of the inferior longitudinal fasciculus, and a part of the left parahippocampus. The regional patterns of increased DR (in red color) were similar to the pattern of FA reduction but in a larger region of the left temporal white 


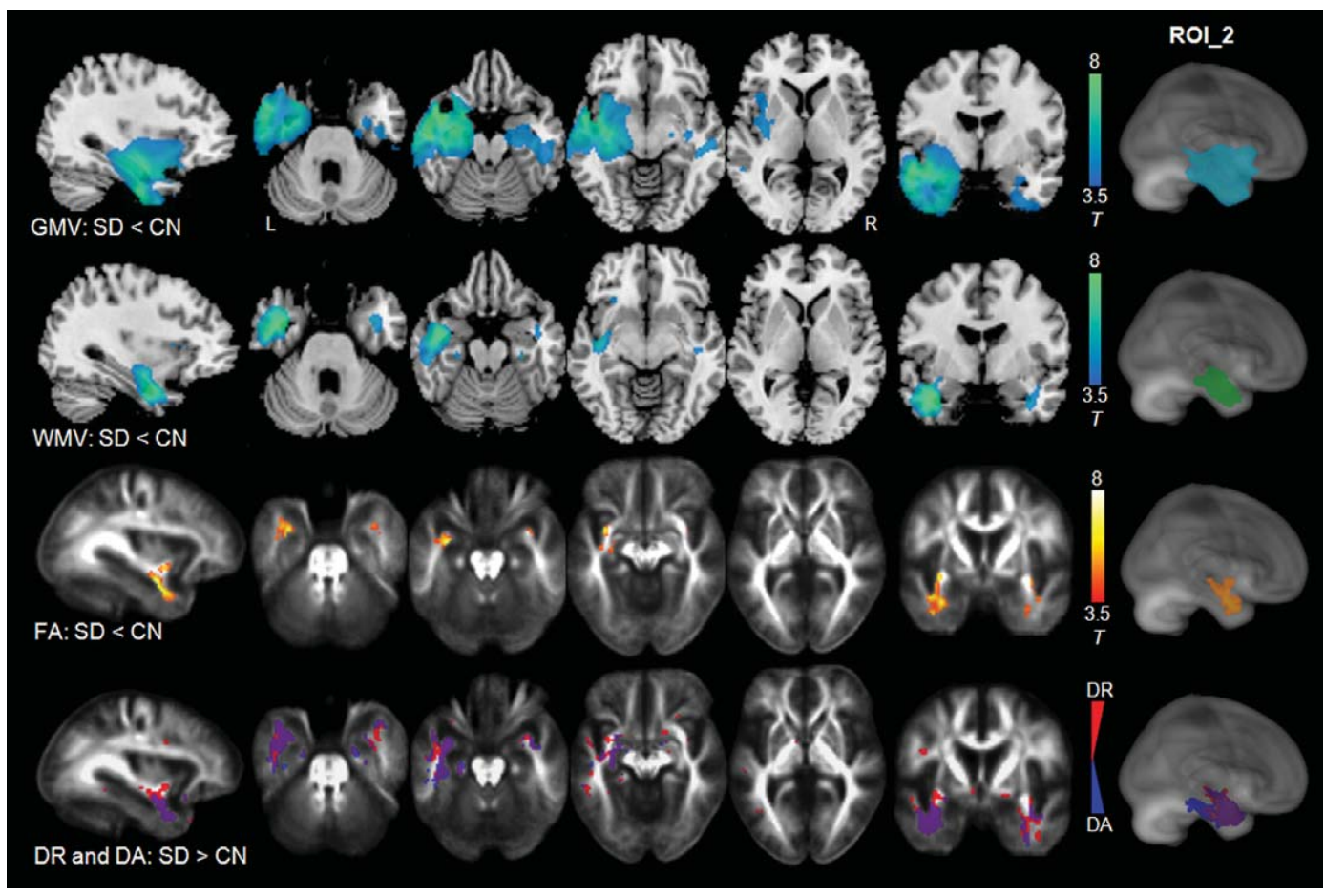

Fig. 2. Comparison between SD and CN, row 1-2: regions of significant gray and white matter atrophy (in blue-green color) superimposed on the T1-weighted image; row 3: the regions of significant FA reduction (in red-yellow color) superimposed on the FA template; row 4: regions of significantly increased DR (in red color) and increased DA (in blue color) superimposed on the FA template. Statistical map-derived ROI was determined by the largest-sized cluster that showed significant $(p=0.001)$ on each paired-group comparison. ROI 2 indicates the anatomical locations of these statistical map-derived ROIs superimposed on a rendered brain.

matter. Increased DA was largely overlapped with the increased DR (in purple color).

\section{Voxel-wise comparisons between PNFA and CN}

Figure 3 shows the pattern of significant, focal gray matter atrophy (row 1), white matter atrophy (row 2), FA reduction (row 3), and an overlay of increased DR and increased DA (row 4) in patients of PNFA compared to CN. PNFA featured predominantly left hemispheric gray matter atrophy including the left posterior portion of the inferior frontal gyrus, and superior frontal gyrus, as well as bilateral anterior insula, anterior cingulate gyri, and caudate nuclei. White matter atrophy was distributed primarily in a few white matter areas adjacent to the language cortices (including Broca's area at the left inferior frontal gyrus and Wernicke's area at the left superior temporal gyrus), and the left anterior cingulate area. Compared to $\mathrm{CN}$, PNFA patients showed a moderate FA reduction predominantly in the left arcuate fasciculus region, most pronounced in the fronto-parietal component of the left arcuate fasciculus, which was spatially linked to language areas that showed structural atrophy. The increased DR (in red color) was also observed predominantly in the dorsal part of the left arcuate fasciculus region, while the increased DA (in blue and purple colors) was mostly seen the left superior motor pathway and some extra-fiber areas adjacent to the left arcuate fasciculus.

Voxel-wise comparisons among FTLD sub-groups

Analysis of FTLD subtypes is limited because of the small sample size. For completeness, results from the subgroups can be summarized as follows: FTD versus PNFA: bvFTD showed smaller GM and WM volumes than PNFA in bilateral medial frontal cortices as well as reduced FA and increased DR in bilateral frontal forceps of the corpus callosum. FTD versus SD: FTD showed smaller GM volume than SD in bilateral middle frontal cortices, and reduced FA in the left frontal forceps of the corpus callosum, while no other measure yielded significant differences. SD versus PNFA: No significant differences were found with any of the measures. 


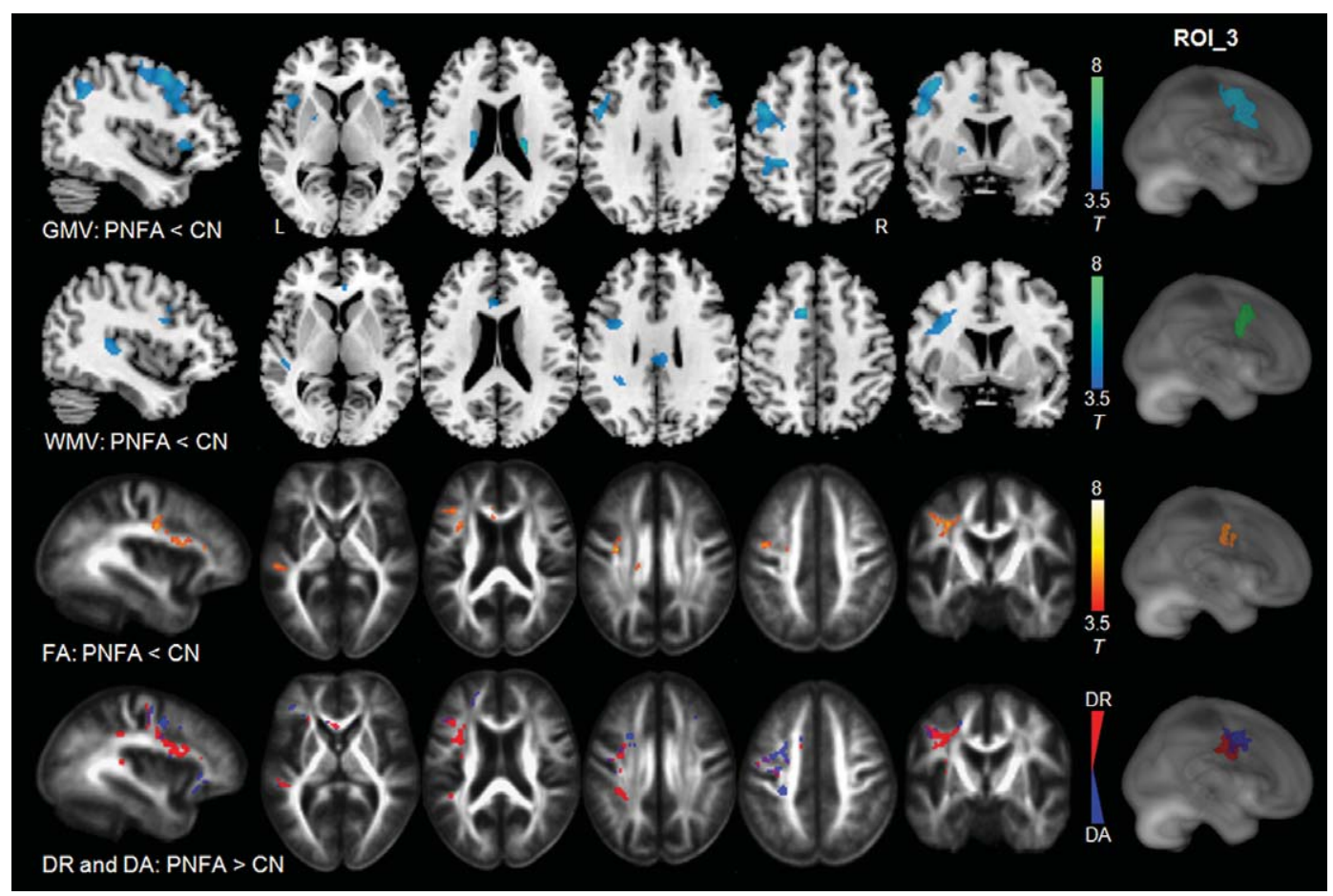

Fig. 3. Comparison between PNFA and CN, row 1-2: gray and white matter atrophy (in blue-green color) superimposed on the T1-weighted image; row 3: the regions of significant FA reduction (in red-yellow color) superimposed on the FA template; row 4: regions of significantly increased DR (in red color) and increased DA (in blue color) superimposed on the FA template. Statistical map-derived ROI was determined by the largest-sized cluster that showed significant $(p=0.001)$ on each paired-group comparison. ROI_3 indicates the anatomical locations of these statistical map-derived ROIs superimposed on a rendered brain.

Cross-modality analyses of volumetric and DTI data in statistical map-derived ROIs

Figure 4 shows the mean and standard deviation of Z-scores from each measurement in each ROI. The ROIs of volumetric measures (gray matter volumes (GMV) and white matter volumes (WMV)) and DTI measures (FA, DR, and DA) derived from the voxelwise group comparisons between bvFTD and $\mathrm{CN}$ were distributed similarly in bilateral frontotemporal lobes and the anterior callosal area (see 'ROI_1' on Fig. 1). ROIs of the five measures derived from the voxelwise group comparison between $\mathrm{SD}$ and $\mathrm{CN}$ were located similarly in the left temporal lobe (see 'ROI_2' Fig. 2), and the ROIs derived from the comparison between PNFA and CN were located in the anatomically associated regions at the left dorsal frontal lobe (see 'ROI_3' Fig. 3). In each set of ROIs, regional GMV, WMV, and DTI (FA, DR and DA) values were significantly correlated with each other across all subjects: Pearson's correlation coefficient $r=0.38-0.87$, $p \leq 0.01$ in ROI_1; $r=0.49-0.91, p \leq 0.01$ in ROI_2, and $r=0.38-0.82, p<0.02$ in ROI_3. Since the data of each measurement from the $\mathrm{CN}$ group was mean centered and variance standardized to one, the extent of the group differences in each measurement can be appreciated directly from variations of the mean Z-score (Fig. 4). Among each group comparison, measures of DR yielded the overall greater Z-scores than other DTI indices (FA and DA) and variations of GMV and WMV. Furthermore, group differences measured by DR remained significant $(p<0.001)$ after accounting for the variations of GMV and WMV. Conversely, significant group differences measured by GMV or WMV diminished $(p>0.4)$ after accounting for DR variations.

\section{Group classifications by DTI indices}

Accuracy of correct FTLD classification based on DTI indices in ROIs is summarized in Table 2. DR of ROI_1 provided the best classification, while DA of ROI_2 was the worst classifier. In particular, DR of ROI_1 achieved a better classification than FA 

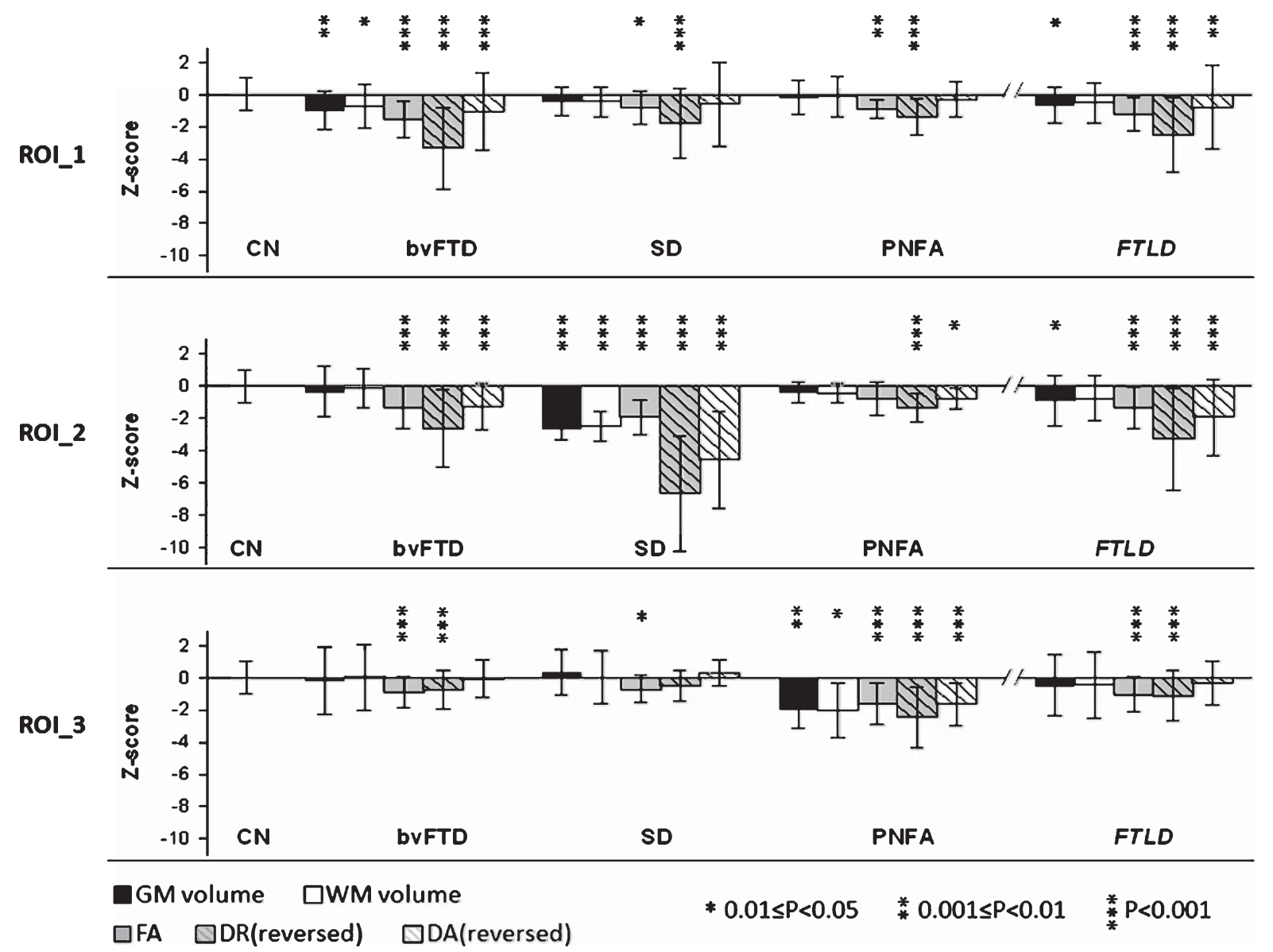

Fig. 4. Mean and standard deviation of GMV, WMV, FA, DR, and DA measures that standardized as Z-scores from three ROI sets derived from the largest cluster shown on the statistical maps. Z-scores of increased DR and DA are reversed to negative scores to be comparable to the other measurements. In the FTLD group, the subtypes are pooled.

or DA of ROI_1 $(p<0.05)$ and also a better classification than DR of ROI_2. Overall, DR achieved a better classification than FA or DA, though this was only a trend $(p=0.08)$. For completeness, classifications achieved with MD are also listed in Table 2.

Group classifications by volumetric and DTI measures

Lastly, we compared the classification accuracies of GMV, WMV, and DTI. The results of the logistic regressions and cross-validation analyses for each set of ROIs with data from the variations of GMV, WMV, and DR are summarized in Table 3. The highest accuracy in separating the diagnostic groups was achieved based on DR (67.6-81.4\%), which was consistently better $(p<0.001)$ than classification based on GMV
(45.7-65.7\% accuracy) or WMV (47.4-59.2\% for accuracy). Classification accuracy of FA (60.5-81.6\%) was also significantly better compared to GMV or WMV. Furthermore, classification accuracies based on GMV of ROI_1 and ROI_2 were better than those based on WMV.

Supplementary results: DTI alterations in tract-specific regions

Supplementary analysis in tract-specific regions was performed for comparisons between each FTLD subtype (bvFTD, SD, and PNFA) and the control group, and among FTLD subtypes. Supplementary Table 1 (available online: http://www.j-alz.com/issues/ 33/vol33-2.html\#supplementarydata05) lists the mean and standard deviation of FA, DA, and DR values in 
Table 2

Mean and standard deviation of Matthews correlation coefficients from a classification between FTLD and controls using nested logistic regressions with cross-validation

\begin{tabular}{|c|c|c|c|c|c|}
\hline Measure/model & Full & ROI_1 & ROI_2 & ROI_3 & Overall mean \\
\hline FA & $0.49 \pm 0.20$ & $0.52 \pm 0.18$ & $0.45 \pm 0.19$ & $0.52 \pm 0.17$ & $0.49 \pm 0.18$ \\
\hline DR & $0.57 \pm 0.18$ & $0.68 \pm 0.16^{\mathrm{a}, \mathrm{b}, \mathrm{c}}$ & $0.48 \pm 0.18$ & $0.61 \pm 0.18$ & $0.61 \pm 0.18$ \\
\hline DA & $0.47 \pm 0.24$ & $0.42 \pm 0.20$ & $0.40 \pm 0.22$ & $0.52 \pm 0.21$ & $0.45 \pm 0.21$ \\
\hline MD & $0.52 \pm 0.22$ & $0.60 \pm 0.19$ & $0.41 \pm 0.19$ & $0.48 \pm 0.19$ & $0.50 \pm 0.19$ \\
\hline
\end{tabular}

Bold: Significant differences $(p<0.05)$ by Wilcoxon signed rank tests.

${ }^{\text {a }}$ Compared to FA and DA of ROI_1.

${ }^{\mathrm{b}}$ Compared to DR of ROI_2.

${ }^{\mathrm{c}}$ Compared to DA of ROI_2 (DA was the worst classification).

Table 3

Classification of FTLD and controls based on DR, GMV, and WMV measures from various ROIs using logistic regression and area under a receiver operator characteristic curve (AUC). Specificity, sensitivity, and accuracy were evaluated using 4-fold cross-validations of the logistic regression results. $p$ values are adjusted by false discovery rate and indicate the significance of the logistic regressions

\begin{tabular}{|c|c|c|c|c|c|}
\hline Measures & Sensitivity (\%) & Specificity (\%) & Accuracy $(\%)$ & AUC & $p$ \\
\hline \multicolumn{6}{|c|}{ Measures in ROI_1 } \\
\hline GMV & $80.1 \pm 7.0$ & $48.7 \pm 8.2$ & $65.7 \pm 4.0^{b}$ & 0.655 & 0.004 \\
\hline WMV & $77.2 \pm 7.2$ & $34.6 \pm 7.6$ & $59.2 \pm 4.1$ & 0.627 & 0.03 \\
\hline DR & $79.9 \pm 6.8$ & $72.3 \pm 9.8$ & $76.0 \pm 4.2^{\mathrm{a}}$ & 0.853 & $<0.001$ \\
\hline \multicolumn{6}{|c|}{ Measures in ROI_2 } \\
\hline GMV & $77.0 \pm 7.0$ & $46.6 \pm 8.9$ & $63.9 \pm 4.5^{\mathrm{b}}$ & 0.722 & 0.001 \\
\hline WMV & $71.5 \pm 7.7$ & $36.4 \pm 6.6$ & $58.1 \pm 3.3$ & 0.657 & 0.002 \\
\hline DR & $80.7 \pm 8.4$ & $80.5 \pm 9.8$ & $81.4 \pm 3.3^{a}$ & 0.877 & $<0.001$ \\
\hline \multicolumn{6}{|c|}{ Measures in ROI_3 } \\
\hline GMV & $74.2 \pm 9.5$ & $5.4 \pm 3.1$ & $45.7 \pm 4.2$ & 0.566 & 0.22 \\
\hline WMV & $79.8 \pm 8.2$ & $5.3 \pm 2.9$ & $47.4 \pm 4.1$ & 0.606 & 0.24 \\
\hline DR & $73.3 \pm 6.9$ & $58.6 \pm 8.6$ & $67.6 \pm 4.1^{\mathrm{a}}$ & 0.722 & $<0.001$ \\
\hline
\end{tabular}

Bold: Differences in classification accuracy between modalities were significant by Wilcoxon signed rank tests. ${ }^{a}$ DR showed better accuracy compared to GMV and WMV.

${ }^{\mathrm{b}}$ GMV showed better accuracy compared to WMV.

each TOI region, and the significance of pair-wise differences. In general, the findings of DTI alteration in tract-specific regions were consistent with results from the voxel-wise analysis, with the exception of a few regions such as the right anterior cingulum and the right arcuate fasciculus where more significant effects were noted in the voxel based analysis compared to the tract-specific analysis. Supplementary Table 2 summarizes sensitivity, specificity, accuracy, and AUC of group classification from all TOIs of FA, DR, and DA measures. DR achieved the best crossvalidated accuracies compared to FA or DA $(p<0.01$ by Wilcoxon signed rank tests). Furthermore, higher accuracy of DR was largely driven by a better specificity compared to the other DTI measures while the sensitivity of DR was not the best. These findings are consistent with the results from statistic-map derived ROI analyses and indicate that DR is a more accurate measure than the other DTI indices.

\section{DISCUSSION}

The major findings of this study are: first, each clinical FTLD subtype is associated with a distinct pattern of microstructural white matter damage, which mirrors the pattern of regional volume loss; second, DR is a more sensitive index for the classification of FTLD patients than FA, DA, or MD; third, DTI achieves overall greater classification power for differentiating FTLD patients from controls than MRI measurements of brain atrophy.

\section{Patterns of FA reduction and brain atrophy in FTLD subtypes}

The finding of a frontotemporal predominance of FA changes in the bvFTD group replicates previous DTI results [17-20]. The pattern of FA reduction also closely parallels the pattern of volume loss in bvFTD, 
as previously reported $[2,6]$. The similarity between the patterns of FA and volume alterations suggest that white matter microstructural alterations in bvFTD are involved directly in the degradation of cortico-cortical networks [30], which are important for behavior, emotion, as well as cognitive functions [31].

In contrast to bvFTD, SD was associated with left temporal predominance of FA alterations, in keeping with the distribution of structural atrophy in the same patients. The FA reductions in the uncinate fasciculus and the anterior part of the left inferior longitudinal fasciculus are consistent with previous reports [18, 20-22]. These findings suggest that the degeneration of the uncinate fasciculus and the anterior inferior longitudinal fasciculus which interconnects the anterior temporal cortex to the orbitofrontal cortex and to occipital cortex, respectively, may play an important role in word retrieval, semantic associations, and aspects of naming that require connections from temporal to frontal components of the language network (e.g., the naming of actions) [32,33]. In addition, our study also revealed FA and diffusivity alterations in the parahippocampal region, which is closely associated with semantic memory, is expected to be affected in SD [34]. We did not, however, observe significant FA abnormalities in other tracts such as the anterior callosum and arcuate fasciculus. This could be due to the small sample size or relatively mild impairment of the patients as evidenced by low CDR scores. Notably, previous reports [21,23] of FA reductions in these fiber tracts (anterior callosum and arcuate fasciculus) have not been consistently seen in SD patients, suggesting these tracts may not be characteristically implicated in semantic dysfunction.

The finding that FA was primarily reduced in the left arcuate fasciculus in PNFA patients is consistent with recent reports from other DTI studies [18, 20, 21]. Agrammatism and effortful speech are the core clinical features of PNFA [13]. The arcuate fasciculus, which links the cortices of the perisylvian language circuitry [35], has consistently been implicated in grammatical speech [36]. Our structural analyses showed volume loss in the left perisylvian region in PNFA, consistent with previous structural imaging studies [9]. The close links between arcuate fasciculus and speech fluency is also supported by a study [37] which reported that lesions in the superior longitudinal fasciculus result in Broca's aphasia, which features non-fluent, effortful, and slow speech. It is well known that different tracts subserve different aspects of language function and that the dorsal part of the arcuate fasciculus is important for fluency, while the left temporal fibers, i.e., the inferior longitudinal fasciculus and the uncinate fasciculus are important for semantic deficits [38]. It appears that atrophy in the perisylvian cortices together with degeneration of the dorsal arcuate fasciculus are key features of PNFA.

\section{Comparison between DTI indices for FTLD classification}

We found DR is a more sensitive index for classifying FTLD than FA, DA, or MD. The result is consistent with another DTI study [22] which focused on semantic dementia. Although FA is frequently used for quantifying white matter damage in neurodegenerative diseases, there is growing evidence that using DR can result in better classification [39]. A DR increase has also been associated specifically with a greater degree of myelin degeneration in animal models in contrast to FA, which by definition is impacted by concordant radial and axial diffusivity variations [39, 40]. This increased DR is likely associated with the histopathological findings of white matter myelin loss that are seen in FTLD. However, DR changes in human brain ought to be interpreted with caution since clear evidence for the biological underpinning is still in question and DTI measurements are fundamentally limited by structural heterogeneity, for example, in low anisotropic voxels such as inclusion of grey matter tissue, crossing fibers or residual misalignment, changes of absolute diffusivities, or eigenvalues do not necessary reflect underlying pathologies [41]. Furthermore, because of the small sample size, classifications of the individual FTLD variants were not reliable and only a pooled sample could be analyzed. It is therefore possible that DR is not the most sensitive measure in general for FTLD, but sensitivity may vary across the different FLTD subtypes. Despite these limitations, our findings suggest DR is a more powerful index to assess white matter damage in FTLD than FA.

\section{Comparison between DTI and structural measures for FTLD classification}

Studies of cortical dementias have focused on detecting changes in gray matter. FTLD shows extensive gray matter damage, but there is also extensive white matter damage detected by DTI as shown in this study and others [16-18]. The relative contribution of gray matter atrophy measured by structural MRI and white matter microstructural changes measured by DTI is not clear. A new finding of this study is that white matter microstructural alterations measured by DTI (especially by DR and FA) were 
greater than that measured by macrostructural volume loss, and therefore DTI had greater classification accuracy for diagnosing FTLD compared with the structural MRI measurement. Furthermore, although the white matter microstructural integrities and cortical volumes are greatly correlated in the frontal brain, our findings demonstrate that DTI measurements in the frontal and temporal regions remain significant in diagnosing FTLD group after correcting for local gray matter atrophy, suggesting that DTI contributes unique diagnostic information beyond that contributed by measuring cortical atrophy. These results are consistent with the view that the white matter pathology in FTLD may be more prominent than the gray matter pathology [42]. The most common etiologies underlying FTLD are tauopathies or TDP-43 immunoreactive inclusions in neurons and glial cells. White matter regions affected by inclusions were found to have marked myelin and axonal loss, as well as abundant gliosis [43, 44]. These microstructural alterations can be sensitively detected by using DTI. Unlike Alzheimer's disease and mild cognitive impairment, in which white matter pathologies such as oligodendroglial apoptosis, myelin or axonal degenerations are often considered to occur secondarily to the neuron loss in cortices, and white matter alterations detected by DTI are usually to a less extent or magnitude than that detected by measuring gray matter atrophy $[45,46]$, white matter pathologies in FTLD may be a predominant event since the tau- or TDP- inclusions are observed directly in white matter [47, 48]. A recent pathological study further suggests that white matter pathology is a major feature of FTLD [49]. On the other hand, since the frontal lobe has abundant of white matter which contains highest proportion of axons connecting to any brain lobe, it appears that white matter integrity in the frontal lobe is vulnerable to demyelinating [50] and small-vessel cerebrovascular disease [51] relative to the rest lobes of the brain. This feature implies that disorders which predominantly affect frontal lobes (such as FTLD, psychiatric disorders, etc.) are worth studying with DTI. A generalization of the findings needs to be considered with caution since more sophisticated algorithms for volume measurements than SPM8 as well as novel diffusion imaging methods, such as diffusion spectrum imaging, might achieve better sensitivity. Nonetheless, our results suggest that neither volume nor DTI information should be ignored for a comprehensive characterization of FTLD and its variants.

This study has several limitations that should be mentioned. First, the diagnosis of FTLD subtypes was not confirmed by autopsy. Therefore, it remains unknown whether the different pathologies associated with these disorders, e.g., tau or TDP-43 affect the white matter similarly. Furthermore, some patients with FTLD symptomatology can be found to have Alzheimer's disease pathology at autopsy. Therefore, the DTI patterns of change observed antemortem may represent a mixture of pathologies. Another limitation is the small sample size, which limits the logistic regression models unsuccessful for differing between FTLD subgroups. Further investigations including a larger group of patients and follow-up scans are warranted to confirm the current results. There are also methodological limitations. Because of the small sample size, a further division of the groups into a training set for statistical selection of ROIs and a validation set to avoid overfitting was impractical. Our findings should therefore be interpreted with caution. A repeat of the analysis on separate datasets for statistical ROI selection and validation of classifications is required to confirm our findings. Another limitation is that we used DTI acquisition sequence with only 6 diffusion encoding directions and a moderate $b$ value, while more directions and higher $\mathrm{b}$ values together with sophisticated diffusion modeling [52] can improve the characterization of diffusion, especially in heterogeneous regions with crossing fibers.

In conclusion, this study implies that the magnitude of the microstructural white matter damage exceeded the magnitude of the macrostructural atrophy in FTLD. DTI therefore is a more powerful measure for FTLD classification than atrophy and furthermore, radial diffusivity is more sensitive in assessing white matter damage in FTLD than the other commonly used DTI measures.

\section{ACKNOWLEDGMENTS}

This work was supported by National Institutes of Health grants (P01AG19724, P50 AG23501, P41 RR23953), which were administered by the Northern California Institute for Research and Education, and with resources of the Veterans Affairs Medical Center, San Francisco, California. We also thank Mr. Shannon Buckley for assistance with image processing.

Authors' disclosures available online (http://www.jalz.com/disclosures/view.php?id=1484).

\section{REFERENCES}

[1] Rabinovici GD, Miller BL (2010) Frontotemporal lobar degeneration: Epidemiology, pathophysiology, diagnosis and management. CNS Drugs 24, 375-398. 
[2] Boccardi M, Laakso MP, Bresciani L, Galluzzi S, Geroldi C, Beltramello A, Soininen H, Frisoni GB (2003) The MRI pattern of frontal and temporal brain atrophy in fronto-temporal dementia. Neurobiol Aging 24, 95-103.

[3] Cardenas VA, Boxer AL, Chao LL, Gorno-Tempini ML, Miller BL, Weiner MW, Studholme C (2007) Deformationbased morphometry reveals brain atrophy in frontotemporal dementia. Arch Neurol 64, 873-877.

[4] Chao LL, Schuff N, Clevenger EM, Mueller SG, Rosen HJ, Gorno-Tempini ML, Kramer JH, Miller BL, Weiner MW (2007) Patterns of white matter atrophy in frontotemporal lobar degeneration. Arch Neurol 64, 1619-1624.

[5] Kanda T, Ishii K, Uemura T, Miyamoto N, Yoshikawa T, Kono AK, Mori E (2008) Comparison of grey matter and metabolic reductions in frontotemporal dementia using FDG-PET and voxel-based morphometric MR studies. Eur J Nucl Med Mol Imaging 35, 2227-2234.

[6] Rosen HJ, Gorno-Tempini ML, Goldman WP, Perry RJ, Schuff N, Weiner M, Feiwell R, Kramer JH, Miller BL (2002) Patterns of brain atrophy in frontotemporal dementia and semantic dementia. Neurology 58, 198-208.

[7] Seeley WW (2009) Frontotemporal dementia neuroimaging: A guide for clinicians. Front Neurol Neurosci 24, 160-167.

[8] Amici S, Gorno-Tempini ML, Ogar JM, Dronkers NF, Miller BL (2006) An overview on primary progressive aphasia and its variants. Behav Neurol 17, 77-87.

[9] Gorno-Tempini ML, Dronkers NF, Rankin KP, Ogar JM, Phengrasamy L, Rosen HJ, Johnson JK, Weiner MW, Miller BL (2004) Cognition and anatomy in three variants of primary progressive aphasia. Ann Neurol 55, 335-346.

[10] Josephs KA, Whitwell JL, Knopman DS, Boeve BF, Vemuri P, Senjem ML, Parisi JE, Ivnik RJ, Dickson DW, Petersen RC, Jack CR Jr (2009) Two distinct subtypes of right temporal variant frontotemporal dementia. Neurology 73, 1443-1450.

[11] Peelle JE, Troiani V, Gee J, Moore P, McMillan C, Vesely L, Grossman M (2008) Sentence comprehension and voxel-based morphometry in progressive nonfluent aphasia, semantic dementia, and nonaphasic frontotemporal dementia. J Neurolinguistics 21, 418-432.

[12] Studholme C, Cardenas V, Blumenfeld R, Schuff N, Rosen HJ, Miller B, Weiner M (2004) Deformation tensor morphometry of semantic dementia with quantitative validation. Neuroimage 21, 1387-1398.

[13] Gorno-Tempini ML, Hillis AE, Weintraub S, Kertesz A, Mendez M, Cappa SF, Ogar JM, Rohrer JD, Black S, Boeve BF, Manes F, Dronkers NF, Vandenberghe R, Rascovsky K, Patterson K, Miller BL, Knopman DS, Hodges JR, Mesulam MM, Grossman M (2011) Classification of primary progressive aphasia and its variants. Neurology 76, 1006-1014.

[14] Ogar JM, Dronkers NF, Brambati SM, Miller BL, GornoTempini ML (2007) Progressive nonfluent aphasia and its characteristic motor speech deficits. Alzheimer Dis Assoc Disord 21, S23-S30.

[15] Rohrer JD, Warren JD, Modat M, Ridgway GR, Douiri A, Rossor MN, Ourselin S, Fox NC (2009) Patterns of cortical thinning in the language variants of frontotemporal lobar degeneration. Neurology 72, 1562-1569.

[16] Borroni B, Brambati SM, Agosti C, Gipponi S, Bellelli G, Gasparotti R, Garibotto V, Di Luca M, Scifo P, Perani D, Padovani A (2007) Evidence of white matter changes on diffusion tensor imaging in frontotemporal dementia. Arch Neurol 64, 246-251.

[17] Matsuo K, Mizuno T, Yamada K, Akazawa K, Kasai T, Kondo M, Mori S, Nishimura T, Nakagawa M (2008) Cerebral white matter damage in frontotemporal dementia assessed by diffusion tensor tractography. Neuroradiology 50, 605-611.

[18] Whitwell JL, Avula R, Senjem ML, Kantarci K, Weigand SD, Samikoglu A, Edmonson HA, Vemuri P, Knopman DS, Boeve BF, Petersen RC, Josephs KA, Jack CR Jr (2010) Gray and white matter water diffusion in the syndromic variants of frontotemporal dementia. Neurology 74, 1279-1287.

[19] Zhang Y, Schuff N, Du AT, Rosen HJ, Kramer JH, GornoTempini ML, Miller BL, Weiner MW (2009) White matter damage in frontotemporal dementia and Alzheimer's disease measured by diffusion MRI. Brain 132, 2579-2592.

[20] Agosta F, Scola E, Canu E, Marcone A, Magnani G, Sarro L, Copetti M, Caso F, Cerami C, Comi G, Cappa SF, Falini A, Filippi M (2011) White matter damage in frontotemporal lobar degeneration spectrum. Cereb Cortex, doi: 10.1093/cercor/bhr288

[21] Galantucci S, Tartaglia MC, Wilson SM, Henry ML, Filippi M, Agosta F, Dronkers NF, Henry RG, Ogar JM, Miller BL, Gorno-Tempini ML (2011) White matter damage in primary progressive aphasias: A diffusion tensor tractography study. Brain 134, 3011-3029.

[22] Acosta-Cabronero J, Patterson K, Fryer TD, Hodges JR, Pengas G, Williams GB, Nestor PJ (2011) Atrophy, hypometabolism and white matter abnormalities in semantic dementia tell a coherent story. Brain 134, 2025-2035.

[23] Agosta F, Henry RG, Migliaccio R, Neuhaus J, Miller BL, Dronkers NF, Brambati SM, Filippi M, Ogar JM, Wilson SM, Gorno-Tempini ML (2010) Language networks in semantic dementia. Brain 133, 286-299.

[24] Basser PJ, Pierpaoli C (1996) Microstructural and physiological features of tissues elucidated by quantitative-diffusiontensor MRI. J Magn Reson B 111, 209-219.

[25] Song SK, Yoshino J, Le TQ, Lin SJ, Sun SW, Cross AH, Armstrong RC (2005) Demyelination increases radial diffusivity in corpus callosum of mouse brain. Neuroimage $\mathbf{2 6}$, 132-140.

[26] Agosta F, Henry RG, Migliaccio R, Neuhaus J, Miller BL, Dronkers NF, Brambati SM, Filippi M, Ogar JM, Wilson SM, Gorno-Tempini ML (2009) Language networks in semantic dementia. Brain 133, 286-299.

[27] Neary D, Snowden JS, Gustafson L, Passant U, Stuss D, Black S, Freedman M, Kertesz A, Robert PH, Albert M, Boone K, Miller BL, Cummings J, Benson DF (1998) Frontotemporal lobar degeneration: A consensus on clinical diagnostic criteria. Neurology 51, 1546-1554.

[28] Masutani Y, Aoki S, Abe O, Hayashi N, Otomo K (2003) MR diffusion tensor imaging: Recent advance and new techniques for diffusion tensor visualization. Eur J Radiol 46, 53-66

[29] Baldi P, Brunak S, Chauvin Y, Andersen CA, Nielsen H (2000) Assessing the accuracy of prediction algorithms for classification: An overview. Bioinformatics 16, 412-424.

[30] Seeley WW, Crawford RK, Zhou J, Miller BL, Greicius MD (2009) Neurodegenerative diseases target large-scale human brain networks. Neuron 62, 42-52.

[31] Tartaglia MC, Zhang Y, Racine C, Laluz V, Neuhaus J, Chao L, Kramer J, Rosen H, Miller B, Weiner M (2012) Executive dysfunction in frontotemporal dementia is related to abnormalities in frontal white matter tracts. J Neurol 259, 1071-1080.

[32] Lu LH, Crosson B, Nadeau SE, Heilman KM, Gonzalez-Rothi LJ, Raymer A, Gilmore RL, Bauer RM, Roper SN (2002) Category-specific naming deficits for objects and actions: Semantic attribute and grammatical role hypotheses. Neuropsychologia 40, 1608-1621. 
[33] Mandonnet E, Nouet A, Gatignol P, Capelle L, Duffau H (2007) Does the left inferior longitudinal fasciculus play a role in language? A brain stimulation study. Brain 130, 623-629.

[34] Graham KS, Simons JS, Pratt KH, Patterson K, Hodges JR (2000) Insights from semantic dementia on the relationship between episodic and semantic memory. Neuropsychologia 38, 313-324.

[35] Glasser MF, Rilling JK (2008) DTI tractography of the human brain's language pathways. Cereb Cortex 18, 2471-2482.

[36] Catani M, Mesulam M (2008) The arcuate fasciculus and the disconnection theme in language and aphasia: History and current state. Cortex 44, 953-961.

[37] Dronkers NF, Plaisant O, Iba-Zizen MT, Cabanis EA (2007) Paul Broca's historic cases: High resolution MR imaging of the brains of Leborgne and Lelong. Brain 130, 1432-1441.

[38] Vigneau M, Beaucousin V, Herve PY, Duffau H, Crivello F, Houde O, Mazoyer B, Tzourio-Mazoyer N (2006) Meta-analyzing left hemisphere language areas: Phonology, semantics, and sentence processing. Neuroimage 30, 14141432 .

[39] Acosta-Cabronero J, Williams GB, Pengas G, Nestor PJ (2010) Absolute diffusivities define the landscape of white matter degeneration in Alzheimer's disease. Brain 133, 529539.

[40] Nair G, Tanahashi Y, Low HP, Billings-Gagliardi S, Schwartz WJ, Duong TQ (2005) Myelination and long diffusion times alter diffusion-tensor-imaging contrast in myelin-deficient shiverer mice. Neuroimage 28, 165-174.

[41] Wheeler-Kingshott CA, Cercignani M (2009) About "axial" and "radial" diffusivities. Magn Reson Med 61, 1255-1260.

[42] Zhang Y, Schuff N, Ching C, Tosun D, Zhan W, Nezamzadeh M, Rosen HJ, Kramer JH, Gorno-Tempini ML, Miller BL, Weiner MW (2011) Joint assessment of structural, perfusion, and diffusion MRI in Alzheimer's disease and frontotemporal dementia. Int J Alzheimers Dis 2011, 546871.

[43] Neumann M, Kwong LK, Truax AC, Vanmassenhove B, Kretzschmar HA, Van Deerlin VM, Clark CM, Grossman M, Miller BL, Trojanowski JQ, Lee VM (2007) TDP-43-positive white matter pathology in frontotemporal lobar degeneration with ubiquitin-positive inclusions. J Neuropathol Exp Neurol 66, 177-183.

[44] Schofield E, Kersaitis C, Shepherd CE, Kril JJ, Halliday GM (2003) Severity of gliosis in Pick's disease and frontotemporal lobar degeneration: Tau-positive glia differentiate these disorders. Brain 126, 827-840.

[45] Walhovd KB, Fjell AM, Amlien I, Grambaite R, Stenset V, Bjornerud A, Reinvang I, Gjerstad L, Cappelen T, DueTonnessen P, Fladby T (2009) Multimodal imaging in mild cognitive impairment: Metabolism, morphometry and diffusion of the temporal-parietal memory network. Neuroimage 45, 215-223.

[46] Johnson DK, Barrow W, Anderson R, Harsha A, Honea R, Brooks WM, Burns JM (2010) Diagnostic utility of cerebral white matter integrity in early Alzheimer's disease. Int $J$ Neurosci 120, 544-550.

[47] Englund E, Brun A (1987) Frontal lobe degeneration of nonAlzheimer type. IV. White matter changes. Arch Gerontol Geriatr 6, 235-243.

[48] Zhukareva V, Mann D, Pickering-Brown S, Uryu K, Shuck T, Shah K, Grossman M, Miller BL, Hulette CM, Feinstein SC, Trojanowski JQ, Lee VM (2002) Sporadic Pick's disease: A tauopathy characterized by a spectrum of pathological tau isoforms in gray and white matter. Ann Neurol 51, 730-739.

[49] Kovacs GG, Majtenyi K, Spina S, Murrell JR, Gelpi E, Hoftberger R, Fraser G, Crowther RA, Goedert M, Budka H, Ghetti B (2008) White matter tauopathy with globular glial inclusions: A distinct sporadic frontotemporal lobar degeneration. J Neuropathol Exp Neurol 67, 963-975.

[50] Kemper TL (1994) Neuroanatomical and neuropathological changes during aging and dementia. Oxford University Press Inc., New York.

[51] Pantoni L, Garcia JH (1997) Pathogenesis of leukoaraiosis: A review. Stroke 28, 652-659.

[52] Tuch DS (2004) Q-ball imaging. Magn Reson Med 52, 13581372 . 